\title{
The loss of IL-31 signaling attenuates bleomycin-induced pulmonary fibrosis
}

1 Dan JK Yombo ${ }^{1,2}$, Varshini Odayar ${ }^{1,2}$, Nishant Gupta ${ }^{3}$, Anil G. Jegga ${ }^{2,4}$, and Satish K

2 Madala $^{1,2}$

3 'Division of Pulmonary Medicine, Cincinnati Children's Hospital Medical Center, Cincinnati,

4 Ohio USA.

$5 \quad{ }^{2}$ Department of Pediatrics, University of Cincinnati, College of Medicine, Cincinnati, Ohio

6 USA.

7 3Division of Pulmonary, Critical Care and Sleep Medicine, University of Cincinnati,

8 Cincinnati, Ohio USA.

9 4Division of Biomedical Informatics, Cincinnati Children's Hospital Medical Center,

10 Cincinnati, Ohio USA

12 * Correspondence: Dr. Satish K. Madala, Cincinnati Children's Hospital Medical Center,

13 Division of Pulmonary Medicine, 3333 Burnet Avenue, MLC 2021, Cincinnati, OH 45229. E-

14 mail: satish.madala@cchmc.org

16 Keywords: Interleukin 31, Interleukin 31 receptor alpha, lung, idiopathic pulmonary fibrosis,

17 bleomycin 


\section{Abstract}

21 Idiopathic Pulmonary Fibrosis (IPF) is a severe fibrotic lung disease characterized by 22 excessive collagen deposition and progressive decline in lung function. Th2 $\mathrm{T}$ cell-derived

23 cytokines including IL-4 and IL-13 have been shown to contribute to inflammation and fibrotic

24 remodeling in multiple tissues. Interleukin-31 (IL-31) is a newly identified cytokine that is

25 predominantly produced by CD4 Th2 T cells, but its signaling receptor IL-31RA is primarily

26 expressed by non-hematopoietic cells. However, the potential role of the IL-31-IL31RA axis

27 in pulmonary inflammation and fibrosis has remained largely unknown. To determine the role

28 of IL-31 signaling in pulmonary fibrosis, wildtype, and IL-31RA knockout mice were treated

29 with bleomycin and measured changes in collagen deposition and lung function. Notably, the

30 loss of IL-31 signaling attenuated collagen deposition and lung function decline during

31 bleomycin-induced pulmonary fibrosis. However, the loss of IL-31RA signaling did not affect

32 inflammation in the lungs. The total lung transcriptome analysis showed a significant

33 reduction in fibrosis-associated gene transcripts including extracellular matrix and epithelial

34 cell-associated gene networks. Furthermore, the lungs of human IPF showed an elevated

35 expression of IL-31 when compared to healthy subjects. In support, the percentage of IL-31

36 producing $\mathrm{CD}^{+}{ }^{+} \mathrm{T}$ cells was greater in the lungs and PBMCs from IPF patients compared to

37 healthy controls. Our findings suggest a pathogenic role for IL-31/IL-31RA signaling during

38 bleomycin-induced pulmonary fibrosis. Thus, therapeutic targeting the IL-31-IL-31RA axis

39 may prevent collagen deposition, improve lung function, and have therapeutic potential in

40 pulmonary fibrosis. 


\section{Introduction}

43 Pulmonary fibrosis is a chronic heterogeneous lung disease characterized by an uncontrolled

44 injury and repair process in the lung parenchyma. This process includes excessive deposition

45 of collagen and extracellular matrix (ECM) protein components in the distal areas of the lung

$46 \quad[1 ; 2]$. Patients with severe fibrotic lung diseases including idiopathic pulmonary fibrosis (IPF)

47 develop irreversible decline of lung function characterized by a decrease in forced expiratory

48 volume in one second (FEV1) and forced vital capacity (FVC) in part due to the progressive

49 accumulation of ECM producing myofibroblasts. Currently, the United States Food and Drug

50 Administration (FDA) approved therapies to treat IPF, Nintedanib or Pirfenidone, slow the rate

51 of FVC decline but do not reverse ongoing fibrosis [3; 4]. While the etiology of IPF is not well

52 established, multiple pathways underlying the pathogenesis of pulmonary fibrosis have been

53 identified $[2 ; 5]$. The development of fibrotic lesions, specifically, is regulated by signaling

54 pathways that are driven by multiple pro-fibrotic growth factors, including TGF- $\beta$, TGF- $\alpha$,

55 CTGF, and cytokines such as IL-1 $\beta, \mathrm{IL}-17$, IL-4, and IL-13 [6; 7; 8]. Th2 cytokines IL-4 and

56 IL-13 have been found to play a major role in the pathophysiology of fibrotic diseases and are

57 considered potential therapeutic targets [8]. IL-13 signaling can induce activation of

58 fibroblasts and macrophages to produce ECM and constitutive factors such as proline, which

59 are required for the biogenesis of collagen. IL-4 and IL-13 signal through a type II IL-4 receptor

60 alpha (IL-4Ra); this receptor is expressed in multiple cell types, including macrophages,

61 epithelial cells, and fibroblasts [9].

Interleukin-31 (IL-31) is a recently described cytokine in the gp130/IL-6 cytokine family

63 and is mainly produced by type 2 helper T cells. It plays a major role in the pathogenesis of

64 chronic inflammatory diseases including atopic dermatitis $(A D)$ or eczema $[10 ; 11 ; 12]$. IL-31

65 signaling is mediated through the heterodimeric receptor composed of the IL-31 receptor A 
66 (IL-31RA) and the Oncostatin M receptor $\beta$ (OSMR $\beta$ ). IL-31RA is expressed by various cells

67 including immune cells, epithelial cells, and fibroblasts; these cells can secrete pro-

68 inflammatory cytokines and chemokines following stimulation by IL-31[11; 13; 14; 15; 16].

69 The role of IL-31 in the development of inflammatory diseases including AD has been

70 described and potential therapeutic agents have been generated to target IL-31/LL-31RA

71 signaling $[10 ; 11 ; 17 ; 18]$. Recent studies have shown elevated levels of IL-31 in patients with

72 AD or asthma and these levels have been correlated with disease severity [19;20]. In fibrotic

73 diseases, several IL-6 family of cytokines including IL6 and OSM have been shown to induce

74 pulmonary fibrosis, but the contribution of IL-31/IL-31RA signaling in lung fibrosis has

75 remained unexplored [20; 21]. Our previous work has depicted the effector mechanism of IL-

$764 / \mathrm{IL}-13$ signaling in the pathogenesis of pulmonary fibrosis and the upregulation of IL-31RA

77 via the IL-4Ra/STAT6 signaling axis [8; 22]. However, the role of IL-31/IL-31RA signaling in

78 the pathogenesis of pulmonary fibrosis has not been definitively determined.

The current study aimed to determine the role of IL-31/IL-31RA signaling in the 80 pathogenesis of pulmonary fibrosis using a bleomycin-induced lung fibrosis model and IL-

81 31RA deficient mice. We observed a significant decrease in collagen deposition and the

82 expression of profibrotic genes in the lungs of IL-31RA deficient mice compared to wildtype

83 mice treated with bleomycin. Notably, the loss of IL-31RA signaling was sufficient to attenuate

84 worsening lung function. Further, we observed increased expression of IL-31 in the lung

85 tissue and CD4-positive T cells of IPF patients compared to healthy subjects. 
Mice. IL31RA knockout (IL-31RA ${ }^{-/}$) mice and their littermates wildtype (WT) mice of C57BL/6

background were used in this study to investigate the role of IL-31RA signaling in the development of pulmonary fibrosis [11]. Both male and female mice at 10-18 weeks of age

were used for all of the experiments and were housed in the Cincinnati Children's Hospital

Medical Center animal facility under conditions approved by the American Association for the

Accreditation of Laboratory Animal Care. All mice were maintained under aseptic conditions

and received sterile food and water. The experiments were approved by the Institutional

Animal Care and Use Committee.

Bleomycin-induced pulmonary fibrosis model. IL-31RA ${ }^{-/-}$and littermate wildtype mice were intradermally injected with bleomycin to induce lung fibrosis as previously described [8; 23]. Briefly, mice were injected with saline or bleomycin ( $6 \mathrm{U} / \mathrm{kg}$ of body weight) in $50 \mu \mathrm{l}$ saline, once a day and during 5 consecutive days per week, for a total of 4 weeks, to induce pulmonary fibrosis. Mice were euthanized on day 28 and lung samples were collected to assess fibrosis using biochemical and molecular methods.

Histology and lung hydroxyproline. The lungs were fixed with $10 \%$ neutral formalin and paraffin embedded. The five-micron thick lung sections were prepared and stained with

104 Masson's Trichome to evaluate the deposition of collagen in lung tissues, a key feature of the

105 fibrotic process. The deposition of collagen was assessed by measuring hydroxyproline levels

106 in lung lysates using a colorimetric detection assay as previously described [24]. In brief, the 107 right lung lobes were collected, weighed, and hydrolyzed in $4 \mathrm{ml}$ of $6 \mathrm{~N} \mathrm{HCl}$ overnight at

$108110^{\circ} \mathrm{C}$. Hydrolyzed samples were neutralized with $1 \mathrm{~N} \mathrm{NaOH}$. For colorization, chloramine-T

$109(0.05 \mathrm{M})$ and the aldehyde-perchloric acid reagent were added and samples were placed in

110 a hot water bath at $60^{\circ} \mathrm{C}$ for $25 \mathrm{~min}$. The concentration of hydroxyproline in the samples was

111 determined using the standard curve. The hydroxyproline level was normalized to the lung 
112 weight and expressed in comparison to the level measured in the saline-treated wildtype

113 control group [8].

114 Lung function measurements. Murine lung function was measured using a computerized

115 FlexiVent system (Flexiware version 7.5, SCIREQ, Montreal Canada) as previously described

116 [25; 26; 27]. Briefly, mice were anesthetized with Ketamine/Xylazine and an incision was

117 performed on the anterior area of the neck to expose the trachea. The trachea was then

118 cannulated with a $20 \mathrm{G}$ cannula and the mouse was connected to the FlexiVent system.

119 Following deep inflation, three consecutive measures were collected for each mouse and the

120 average value was used to measure resistance, elastance, and compliance.

121 Immunohistochemistry. Formalin-fixed and paraffin-embedded human lung tissue sections

122 of IPF $(n=8)$ and healthy $(n=7)$ controls were immunostained with antibodies against IL-31 as

123 previously described [28; 29]. Briefly, rabbit anti-human IL-31 monoclonal antibody (R\&D

124 Systems) was used as the primary antibody (1:25 dilution). Goat anti-rabbit lg was conjugated

125 with a peroxidase enzyme to form a brown precipitate in the presence of hydrogen peroxide

126 and DAB. Nuclei were stained in blue using Hematoxylin counterstain as described in

127 previous reports [23; 30]. The staining of the lung section using control IgG showed no

128 detectable immunostaining (data not shown). All images were obtained using a Leica

129 DM2700 M bright-field microscope. High-magnification images (X40) were captured with a

1303 3CCD color video camera. The number of IL-31-positive cells and total cells was counted

131 using MetaMorph imaging software and expressed as the percentage of IL-31-positive cells

132 in total cells in the field.

133 RNA preparation and real-time PCR. Lungs were homogenized using Qiagen tissue 134 homogenizer and beads (Qiagen), and total RNA was extracted from lung tissues using 
135 RNeasy mini kit (Qiagen Science, Valencia, CA) as previously described [10; 22]. Lung RNA

136 samples were reverse transcribed into cDNA using Superscript III (Invitrogen), and the

137 relative transcript expression of select genes was measured using SYBR Green PCR Master

138 Mix (Applied Biosystems, Foster City, CA) and the CFX384 Touch Real-Time PCR detection

139 system (Bio-Rad, Hercules, CA) as previously described [31]. Gene transcripts were

140 normalized to the housekeeping gene hypoxanthine-guanine phosphoribosyltransferase

141 (HPRT) or $\beta$ actin and were expressed as the relative fold-induction change compared to the

142 gene expression level of the control wildtype mice treated with saline solution. Data was

143 analyzed with Bio-Rad CFX maestro software version 4.2(Bio-Rad Laboratories, Hercules,

$144 \mathrm{CA})$. The list of primers used for real-time PCR to analyze the relative expression of the genes

145 is included in Supplementary Table 1.

146 Whole lung transcriptome shotgun sequencing (RNAseq) Total lung RNA samples were

147 obtained from IL-31RA ${ }^{-/-}$and wildtype mice treated with bleomycin as described above. Four

148 samples were prepared from each experimental group and subjected to RNA sequencing

149 using an Illumina HiSeq-1000 Sequencer (Illumina, San Diego, CA), as described previously

150 [32]. A comparative analysis between groups was realized to identify genes with differential

151 changes in expression following treatment with bleomycin or saline. Genes with statistically

152 significant changes were selected based on a $p$-value cut-off of 0.05 and differential

153 expression (defined as a 1.5-fold increase or decrease in expression). Heatmaps of genes

154 with differential expression change were generated to highlight clusters of genes up-or

155 downregulated in IL-31RA ${ }^{-/-}$and wildtype mice treated with bleomycin. Functional enrichment

156 analyses were performed using the ToppFun application of the ToppGene Suite [33]. The IL-

157 31RA-dependent genes were also compared with differentially expressed genes (DEGs) from 
158 IPF patients (GSE53845) to identify common genes between IL-31RA regulated genes and

159 IPF DEGs as described previously [34; 35].

160 In vitro epithelial cell treatments. Human bronchial epithelial BEAS-2B cells were obtained

161 from the American type culture collection (Manassas, VA) and cultured in DMEM medium

162 supplemented with $10 \%$ fetal bovine serum (FBS) at $37^{\circ} \mathrm{C}$ and $5 \% \mathrm{CO}_{2}$. To assess the effects

163 of IL-31, airway epithelial cells were seeded in 12 or 24 well plates in low serum media

164 (1\%FBS) for $16 \mathrm{hrs}$. Under low serum conditions, cells were stimulated with media

165 supplemented with increasing doses $(0,100,250$, or $500 \mathrm{ng} / \mathrm{ml})$ of recombinant IL-31(R\&D

166 Systems) for 18 hours. Total RNA was extracted from the cultured cells using the Qiagen

167 RNA shredder and the RNeasy mini kit (Qiagen). cDNA was prepared and applied for real-

168 time RT-PCR to measure gene transcript levels as described above. Gene expression was

169 normalized to the housekeeping gene human $\beta$-actin.

170 Flow Cytometry. Peripheral blood mononuclear cells (PBMC) were isolated from peripheral

171 venous blood of IPF patients $(n=11)$ and healthy control donors $(n=10)$ using Ficoll-paque

172 density gradient-based separation with centrifugation (GE Healthcare, Uppsala, Sweden).

173 Red blood cells were lysed using an ACK lysis buffer and cell viability was confirmed with

174 trypan blue. Isolated PBMCs were frozen with DMSO and stored in liquid nitrogen until used.

175 To measure the production of IL-31 and IL-4, PBMCs were thawed from liquid nitrogen and

176 plated in a 96-well round-bottom plate $\left(1 \times 10^{6}\right.$ cells/well) in RPMI 1640 medium

177 supplemented with L-glutamine, antibiotics (penicillin/streptomycin), and 10\% heat-

178 inactivated fetal bovine serum (FBS). Cells then were allowed to rest overnight. PBMCs were

179 stimulated with phorbol 12-myristate 13-acetate (PMA) $10 \mathrm{ng} / \mathrm{ml}$ with lonomycin (1ug/ml), and

180 Golgi stop (1:1500) for 5 hours to induce cytokine production. Control samples were cultured

181 with PMA and ionomycin for the same period. Cells were then stained with Zombie 
$182 \mathrm{UV}$ (BioLegend) for $30 \mathrm{~min}$ at room temperature in the dark to allow for discrimination between

183 living and dead cells. Non-specific binding of fluorochromes was prevented by incubating cells

184 with Human BD Fc Block ${ }^{\mathrm{TM}}$ (BD Biosciences), followed by surface marker staining with anti-

185 CD3-V500 (UCHT1) and anti-CD4-APC(RPA-T4) (Bio Legend). Cells were then fixed and

186 permeabilized using Cytofix/Cytoperm (BD Biosciences) and stained with antibodies against

187 IL-31-PE (U26-947) (BD Biosciences) and IL-4 -PE-Cy7 (MP4-25D2) (BioLegend). Data was

188 acquired using LSR Fortessa flow cytometer and analyzed using FlowJo 10 (FlowJo, Tree 189 Star).

190 Statistical analysis. All data were analyzed using GraphPad PRISM 8. One-way ANOVA 191 with Turkey's multiple comparison test was used to compare different experimental groups.

192 Student's $t$ test was used to compare between two experimental groups. Quantitative data 193 were presented as mean \pm SEM, and $p<0.05$ was considered statistically significant.

\section{Results}

196 The loss of IL-31RA signaling attenuates collagen deposition and lung function decline

To determine whether the loss of IL-31RA has any effect on pulmonary fibrosis, IL-

198 31RA knockout mice and their littermate wildtype mice were treated intradermally with bleomycin (to induce pulmonary fibrosis) or treated with saline as a non-fibrosis control. To assess collagen deposition, the lung sections were stained with Masson Trichrome. There

201 were no significant differences in collagen staining between wildtype and IL-31RA knockout

202 mice treated with saline. A significant increase in collagen deposition was observed in the 203 lungs of both wildtype mice and IL-31RA knockout mice treated with bleomycin (Figure 1A). 
204 However, there was a modest decrease in collagen staining in mice deficient for IL-31RA

205 compared to wildtype mice treated with bleomycin (Figure 1A). To further evaluate collagen

206 deposition, the level of hydroxyproline was quantified in the lungs of IL-31RA knockout mice

207 and wildtype mice treated with bleomycin or saline. Both wildtype and IL-31RA knockout mice

208 treated with bleomycin had increased expression of lung hydroxyproline compared with

209 saline-treated mice. However, there was a modest but significant reduction in lung

210 hydroxyproline levels in IL-31RA knockout mice compared to wildtype mice treated with

211 bleomycin (Figure 1B). Our published studies have demonstrated that repetitive bleomycin-

212 induced fibrotic remodeling decreases lung function [36; 37]. Therefore, we assessed whether

213 the loss of IL-31RA signaling has any effect on the decline in lung function following repetitive

214 bleomycin treatment. Consistent with previous findings, there was an increase in resistance,

215 and elastance, and a significant decrease in compliance in wildtype mice treated with

216 bleomycin compared to saline-treated wildtype or IL-31RA knockout mice (Figure 1C-1E).

217 However, the decline in lung function was attenuated with the loss of IL-31RA signaling

218 compared to wildtype mice treated with bleomycin (Figure 1C-1E). These findings suggest

219 that the loss of IL-31RA has a partial protective effect against collagen deposition, and the

220 subsequent decline in lung function, during bleomycin-induced pulmonary fibrosis.

221 The role of IL-31RA signaling in fibrosis-associated gene expression

To identify IL-31RA dependent gene expression and associated signaling pathways

223 involved in bleomycin-induced pulmonary fibrosis, whole lung transcriptome analysis was

224 performed using next-generation RNA-seq analysis in wildtype and IL-31RA knockout mice

225 treated with bleomycin. The comparison of differentially expressed genes, with 1.5 -fold up-or

226 down-regulation in wildtype and IL31RA knockout lungs, highlighted two clusters of genes as

227 illustrated in the heatmap (Figure 2). A total of 458 genes were differentially expressed in the 
228 lungs of IL-31RA knockout mice compared to wildtype mice treated with bleomycin. About

229228 genes were up-regulated in IL-31RA knockout mice, and 230 genes were down-

230 regulated, with the loss of IL-31RA signaling (Supplementary Table 2). Gene ontology

231 enrichment analysis of down-regulated genes suggested IL-31RA is involved in the

232 expression of several ECM-associated genes (Col1a1, Col3a, Fn1, Arg1, Timp1, Mmp13,

233 Ereg, and IL-6), Protein G coupled receptor Ga(i) signaling (Ccl28, Rgs5, Rgs4, and Rgs16),

234 Epithelium-associated genes (Krt4, Krt5, Krt14, Krt20), Adenylate cyclase activity (Calcb,

235 Calcr) and chemotaxis (Ccl28, Mcp1, IL6). Enrichment analysis of genes that were

236 upregulated with the loss of IL-31RA suggested an increase in the expression of genes

237 associated with the G protein-coupled receptor, and synaptic and calcium signaling.

238 Next, to identify the IL-31RA-dependent genes that are also dysregulated and relevant

239 to IPF pathogenesis, differentially expressed genes in IPF lungs were obtained and compared

240 to IL-31RA-dependent genes (Figure 2B). In total, 181 IPF differentially expressed genes

241 were identified that were also dysregulated with the loss of IL-31RA (Figure 2B). This IL-31RA

242 dependent gene list includes 31 genes that were up-regulated, and 46 down-regulated genes,

243 in IPF lungs (Figure 2B and Supplementary Figure 1). We performed an enrichment analysis

244 of these negatively correlated genes using the ToppFun application and visualized the gene

245 networks using Cytoscape. As shown in Figure 2C, our gene functional enrichment analysis

246 further supports alterations in fibroblast- and epithelial cell-associated genes and also a

247 decrease in multiple collagen gene transcripts.

248 The loss of IL-31RA signaling attenuates the expression of pro-fibrotic genes

To validate the findings using RNA-seq, fibrosis-associated gene transcripts were 250 quantified in the total lung RNA of IL-31RA knockout and wildtype mice treated with bleomycin 
or saline. The major ECM gene transcripts including Col1 $\alpha$, Col3 $\alpha$, and Fn1 were upregulated

252 in lungs of wildtype mice treated with bleomycin compared to wildtype mice treated with

253 saline. This increase in ECM gene expression was significantly attenuated in IL-31RA

254 knockout mice compared to wildtype mice treated with bleomycin (Figure 3A). Similarly,

255 genes associated with ECM production and remodeling were significantly decreased with the

256 loss of IL-31RA; these genes included MMP13 and TIMP1, as well as IL-6 which increased

257 in wildtype mice during bleomycin-induced pulmonary fibrosis (Figure $3 \mathrm{~B}$ ). The total lung

258 RNA-seq analysis also suggests altered expression of several epithelial cell-associated

259 genes with the loss of IL-31RA during bleomycin-induced pulmonary fibrosis (Figure 2). To

260 validate the differential expression of genes associated with epithelial cells, we measured

261 transcript levels of several epithelium-associated genes including Krt5, and Krt14. As shown

262 in Figure 4A, we observed a significant increase in the transcripts of Krt5, and Krt14 in

263 wildtype mice treated with bleomycin compared to saline-treated wildtype mice. This increase

264 in epithelial gene transcripts was attenuated with the loss of IL-31RA compared to wildtype

265 mice treated with bleomycin (Figure 4A). These findings suggest a critical role for airway

266 epithelium in the pathophysiology of IL-31RA-driven pulmonary fibrosis and decline in lung

267 function. Therefore, we studied the effect of IL-31 on airway epithelial cells in the production

268 of pro-fibrotic cytokines associated with pulmonary fibrosis. Bronchial epithelial cell line

269 BEAS-2B, or primary bronchial epithelial cells, were stimulated with IL-31 cytokine and the

270 transcript levels of Mcp1 and IL-6 were determined using RTPCR. The production of pro-

271 fibrotic cytokines, including Mcp1 and IL-6, increased with IL_31 stimulation in both BEAS-

272 EB and HBEC cells (Fig. 4B \& 4C). These findings suggest that IL-31-driven signaling

273 increases the production of pro-fibrotic cytokines by epithelial cells in the pathogenesis of

274 pulmonary fibrosis. 


\section{Increased frequency of IL-31 producing cells in IPF patients}

the lung sections obtained from healthy controls and IPF patients. In contrast to normal lungs,

278 we observed prominent immunostaining for IL-31 in cells populated in the thickened

279 parenchymal areas of IPF lungs (Figure 5A). We quantified the number of IL-31-positive cells

280 and total cells in lung images to assess the accumulation of IL-31-positive cells in IPF lungs

281 compared to healthy lungs. There was a significant increase in the percent of IL-31-positive

282 cells in the lungs from IPF patients compared with healthy controls (Figure 5B). Previous

283 studies have described type 2 CD4 T cells as the main source of IL-31 production in chronic

284 allergic diseases such as AD $[11 ; 38]$. To determine the frequency of IL-31 producing CD4 T

285 cells in IPF, PBMCs were stimulated with PMA-ionomycin or medium for $6 \mathrm{~h}$ and fixed. Cells

286 were fluorescently stained with IL-31 and IL-4 and analyzed by flow cytometry with a gating

287 strategy as illustrated in Supplementary Figure 2. Notably, the majority of IL-31 producing

288 cells in PBMCs were T cells. CD4 T cells producing IL-31, or IL-4 and IL-31, were significantly

289 increased in PBMCs of IPF patients compared to healthy controls (Figure 5D \& 5E).

\section{Discussion}

In this study, we have demonstrated that bleomycin-induced pulmonary fibrosis is decrease in hydroxyproline and downregulation of several pro-fibrotic gene transcripts. In addition, our findings demonstrate that the loss of IL-31RA attenuates lung function decline

296 as observed during bleomycin induced-pulmonary fibrosis. We also showed that IL-31-

297 positive cells are increased in fibrotic lung lesions of IPF patients and IL-31 is predominantly 
produced by CD4 T cells. These findings illustrate the potential utility of targeting the IL-31/IL31RA axis to treat IPF.

Previous studies have identified important roles for Th2 T cell-derived IL-4 and/or IL-

30113 in the development of pulmonary fibrosis and other fibrotic diseases. However, the role of

302 IL-31/IL-31RA signaling in pulmonary fibrosis has remained unexplored. Our previous study

303 along with other research studies have shown that the administration of exogenous IL-31, or

304 overexpression of IL-31, in transgenic mice induces skin thickening and alters the skin barrier.

305 These findings suggest that IL-31/IL-31RA signaling plays an integral role in inflammation and

306 fibrosis [10; 11]. Previous studies have suggested a potential role for IL-31-driven signaling

307 in the development of fibrosis in various organs including the liver [39] and intestine [40].

308 Shish and colleagues reported increased expression of IL-31RA on intestinal myofibroblasts

309 following treatment with TI1a, a TNF superfamily 15 protein, and established an association

310 between IL-31RA signaling and colonic fibrosis [40]. Here, we dissected the pro-fibrotic role

311 of the IL-31/IL-31RA axis using a mouse model of bleomycin-induced pulmonary fibrosis. Our

312 studies which were conducted using IL-31RA knockout mice revealed a significant decrease

313 in hydroxyproline and subsequent improvement in lung function. In addition, whole lung

314 transcriptome analysis revealed a decrease in the expression of ECM-related genes, which

315 thus supports the role of IL-31/IL-31RA in lung fibrosis. Our findings align with a recent

316 publication demonstrating that in scleroderma IL-31 contributes to skin fibrosis; in this study,

317 a sub-cutaneous mini-pump was implanted in the skin to deliver IL-31 [41]. Together, these

318 studies indicate that IL-31-driven signaling is a key factor driving pulmonary fibrosis. However,

319 additional studies are needed to identify specific mechanisms by which IL-31 contributes to

320 ECM production and decline in lung function during bleomycin-induced pulmonary fibrosis. 
322 is consistent with our findings. We demonstrated a significant increase in IL-31 and IL-4/IL-

323 31-producing CD4+ T cells in PBMCs of IPF patients compared with control healthy subjects.

324 The expression of IL-31 was significantly increased in lung tissues of IPF patients compared

325 to control subjects. These findings may indicate that during pulmonary fibrosis development,

326 the $\mathrm{TH}_{2} \mathrm{CD}^{+} \mathrm{T}$ cell population contributes to the increased expression of IL-31 in fibrotic

327 lung lesions; within these lung lesions, IL-31 interacts with the unique receptor IL-31RA that

328 is expressed in stromal cells. Collectively, these observations make a compelling case that

329 hematopoietic cell-derived IL-31 might play a role in the pathogenesis of pulmonary fibrosis

330 by activating non-hematopoietic cells such as epithelial cells and mesenchymal cells. Indeed,

331 stimulation of airway epithelial cells with IL-31 induced an increased production of MCP1 and

332 IL-6 cytokines known to play a role in the development of pulmonary fibrosis [42].

We applied the whole lung transcriptome through RNA seq analysis to identify IL-31/IL-

334 31RA -dependent genes and pathways that might participate in IL-31-driven pulmonary

335 fibrosis. Expression of genes associated with the ECM formation was significantly

336 downregulated in IL-31RA ${ }^{-/-}$mice treated with bleomycin when compared to wildtype mice.

337 Notably, genes associated with epithelial tissue integrity (Krt5 and Krt14), protein Ga

338 signaling, as well as previously reported genes associated with lung function and remodeling

339 (Arg1, Ccl28 and Rgs4) were down regulated in IL-31RA ${ }^{-/-}$fibrotic mice [43; 44; 45].

340 Weathington and colleagues have recently reported an association between increased

341 expression of the genes KCNJ2 and KRT18 in the setting of asthma, suggesting a strong

342 relationship between lung function and tissue remodeling [46]. In support, genes related to

343 membrane potential regulation, including genes for potassium channels Kcna2, Kcnh4, and

344 Kcnu1, were upregulated in IL-31RA ${ }^{-/-}$mice compared to wildtype mice. These findings 
345 support a mechanistic pathway that mediates improved lung function in the absence of IL-

346 31RA signaling. Additional investigation is needed to explore whether IL-31/IL-31RA signaling

347 contributes to the regulation of potassium channel activity and whether altered keratin gene

348 expression in epithelial cells could mediate collagen deposition and lung function.

349 In conclusion, our data indicates that genetic deletion of IL-31RA significantly

350 attenuates collagen deposition and lung function decline induced during repetitive bleomycin-

351 induced injury and pulmonary fibrosis. In recent years, several cytokines have been

352 implicated in the pathogenesis of pulmonary fibrosis. While IL-31 has been identified in

353 several inflammatory and remodeling diseases, mechanistic studies evaluating IL-31

354 producing cells in IPF, and the role of IL-31 in pulmonary fibrosis have been missing. Our

355 results provide new evidence that supports the pathogenic role of IL-31RA-driven signaling

356 in bleomycin-induced pulmonary fibrosis. The IL-31-IL31RA axis may serve as a novel

357 therapeutic target for the treatment of pulmonary fibrosis.

\section{Conflict of Interest}

360 The authors declare that the research was conducted in the absence of any commercial or

361 financial relationships that could be construed as a potential conflict of interest.

\section{Author Contributions}

364 SKM conceived and designed the research. DJY, and SKM performed the experiments and 365 wrote the manuscript. AGJ performed bioinformatic analysis and edited the manuscript. NG 
366 provided human IPF samples and edited the manuscript. VO analyzed data and edited the

367 manuscript.

368

369 Funding

370 This work was supported by NIH [1R01 HL134801, 1R21 Al137309, and W81XWH-17-1-

371 0666) (SKM)].

372

373 Acknowledgments

374 The authors thank Dr. Brijendra Singh, Dr. Edukulla Ramakrishna, and the veterinary

375 services and pathology research core at Cincinnati Children's Hospital Medical Center for

376 help in this study.

378 Data Availability Statement

379 The datasets analyzed in this study can be found here:

380 https://www.ncbi.nlm.nih.gov/geo/query/acc.cgi?acc=GSE53845.

381 https://www.ncbi.nlm.nih.gov/geo/query/acc.cgi?acc=GSE163740.

382

383 Supplementary Table 1. List of primers used for RT-PCR

384 Supplementary Table 2: List of IL-31RA-dependent genes 
386 Figures legend

387 Figure 1. The loss of IL-31RA signaling attenuates collagen deposition and lung 388 function decline during bleomycin-induced pulmonary fibrosis. wildtype (IL-31RA ${ }^{+/+}$) 389 and IL-31RA knockout (IL31RA ${ }^{-/}$) mice were treated intradermally with bleomycin or saline 390 for four weeks to induce pulmonary fibrosis. (A) Lung sections of saline or bleomycin-treated

391 mice were stained with Masson Trichrome. Scale bar $300 \mu \mathrm{m}$. (B) Total lung hydroxyproline 392 was compared between IL-31RA ${ }^{-/-}$and IL31RA ${ }^{+/+}$mice treated with bleomycin or saline. (D-

393 F) Lung function measurements including resistance, elastance and compliance were 394 measured in all four groups using FlexiVent. Data are presented as means \pm SEM. The above 395 data is cumulative of two independent experiments ( $n=12-19 /$ group). Statistical analysis was 396 performed using one-way ANOVA with Tukey's multiple comparisons test. ${ }^{* *} p<0.01$; 397 ${ }^{* * * *} \mathrm{p}<0.001$

Figure 2. The IL-31-IL-31RA axis regulated gene networks during bleomycin-induced pulmonary fibrosis and IPF. Total lung RNA was isolated from bleomycin or saline-treated mice and performed RNA-seq analysis. (A) The heat map shows two clusters of differentially expressed genes that were up-or down-regulated by at least 1.5-fold upon bleomycin 402 treatment of genetic knockdown of IL-31RA compared to wildtype mice. Gene Ontology 403 Enrichment analysis for IL-31RA-dependent gene transcripts was performed using ToppFun. 404 (B) Venn diagram of IL31RA-regulated genes compared with IPF-differentially expressed genes in the lungs of IPF patients. (C) IL-31RA-driven gene networks that are activated in 406 IPF were analyzed using ToppFun and visualized using Cytoscape. Red- and violet-colored 407 oval shapes represent genes that are IL31RA-regulated and up-or down-regulated 
respectively in IPF lungs. Both square and rectangle shapes represent different enriched

biological processes and cell types for the inversely correlated genes between the IL-31-IL-

410 31RA axis and IPF.

411 Figure 3 . The loss of IL-31RA signaling attenuates the expression of fibrosis-

412 associated genes during bleomycin-induced pulmonary fibrosis. Total lung RNA was

413 isolated from wildtype and IL-31RA ${ }^{-/-}$mice treated with saline and bleomycin and fibrosis-

414 associated genes quantified using real-time RTPCR. (A) Quantification of ECM gene

415 transcripts including Col1a, Col3a1, and Fn1. (B ) Quantification of gene transcripts involved

416 in ECM remodeling and production including Mmp13, Timp1, and IL-6. Data presented as

417 Mean \pm SEM ( $n=4 /$ group). Statistical analysis was performed using one-way ANOVA with

418 Tukey's multiple comparisons test ${ }^{*} p<0.05 ;{ }^{* *} p<0.01 ;{ }^{* * *} p<0.005 ;{ }^{* * *} p<0.001$.

419 Figure 4: The loss of IL-31RA signaling alters the expression of epithelial cell-specific 420 gene expression during bleomycin-induced pulmonary fibrosis. (A) Reduced expression

421 of epithelial cell-specific genes including KRT5 and KRT14 in the lungs during bleomycin-

422 induced pulmonary fibrosis. (B) Bronchial epithelial cell line BEAS-2B was stimulated with

423 recombinant IL-31 for 24 hours and the transcripts of MCP1 and IL-6 measured using RT-

424 PCR. (C) primary human bronchial epithelial (NHBEC) cells were stimulated with recombinant

425 IL-31 for 24 hours and the transcripts of MCP1 and IL-6 measured using RT-PCR. Data

426 presented as Mean \pm SEM ( $n=4$ mice/group). Statistical analysis was performed using one-

427 way ANOVA with Tukey's multiple comparisons test ${ }^{*} p<0.05 ;{ }^{* *} p<0.01 ;{ }^{* * *} p<0.005 ;{ }^{* * * *}$ $428 \quad p<0.001$.

429 Figure 5: Increased staining and frequency of IL-31-positive CD4 T cells in IPF. Lung 430 sections from IPF and non-IPF control subjects were probed to detect the expression of IL- 
43131 by immunohistochemistry, (A) Representative images of IL-31 expression in non-IPF (top)

432 and IPF (bottom) lung sections; (B) Percent of IL-31+ cells quantified in IHC stained lung

433 sections. Data are shown as mean \pm SEM., ${ }^{* *} p<0.01$. Peripheral blood mononuclear cells

434 (PBMC) of IPF patients $(n=7)$ and healthy control subjects $(n=8)$ were isolated and stimulated 435 with PMA and ionomycin, intracellular cytokine expression of IL-31, and IL-4 were assessed 436 by flow cytometry. (C) Representative images of CD3 CD4 cells expressing IL-31 and IL-4, 437 unstimulated (top) or stimulated cells with PMA( bottom) from Non-IPF and IPF patients are 438 shown ; (D) Percent of IL-31 and (E) IL4/IL-31- producing CD4 T cells are shown. Data 439 presented as mean \pm SEM with $n=6-9$, with * $p<0.05,{ }^{* *} p<0.01$. Student $t$-test was used to 440 compare the mean between groups.

\section{References}

443 [1] G. Sgalla, B. lovene, M. Calvello, M. Ori, F. Varone, and L. Richeldi, Idiopathic pulmonary fibrosis: pathogenesis and management. Respir Res 19 (2018) 32.

445 [2] P.J. Wolters, H.R. Collard, and K.D. Jones, Pathogenesis of idiopathic pulmonary fibrosis. Annu Rev Pathol 9 (2014) 157-79.

[3] L. Richeldi, R.M. du Bois, G. Raghu, A. Azuma, K.K. Brown, U. Costabel, V. Cottin, K.R. Flaherty, D.M. Hansell, Y. Inoue, D.S. Kim, M. Kolb, A.G. Nicholson, P.W. Noble, M. Selman, H. Taniguchi, M. Brun, F. Le Maulf, M. Girard, S. Stowasser, R. SchlenkerHerceg, B. Disse, H.R. Collard, and I.T. Investigators, Efficacy and safety of (2014) 2071-82. 
[4] T.E. King, Jr., W.Z. Bradford, S. Castro-Bernardini, E.A. Fagan, I. Glaspole, M.K.

Glassberg, E. Gorina, P.M. Hopkins, D. Kardatzke, L. Lancaster, D.J. Lederer, S.D.

Nathan, C.A. Pereira, S.A. Sahn, R. Sussman, J.J. Swigris, P.W. Noble, and A.S.

Group, A phase 3 trial of pirfenidone in patients with idiopathic pulmonary fibrosis. N

[5] V. Sontake, P.R. Gajjala, R.K. Kasam, and S.K. Madala, New therapeutics based on emerging concepts in pulmonary fibrosis. Expert Opin Ther Targets 23 (2019) 69-81.

[6] M.S. Wilson, S.K. Madala, T.R. Ramalingam, B.R. Gochuico, I.O. Rosas, A.W. Cheever, and T.A. Wynn, Bleomycin and IL-1beta-mediated pulmonary fibrosis is IL-17A dependent. J Exp Med 207 (2010) 535-52.

[7] K.K. Kim, D. Sheppard, and H.A. Chapman, TGF-beta1 Signaling and Tissue Fibrosis. Cold Spring Harb Perspect Biol 10 (2018).

[8] B. Singh, R.K. Kasam, V. Sontake, T.A. Wynn, and S.K. Madala, Repetitive intradermal bleomycin injections evoke T-helper cell 2 cytokine-driven pulmonary fibrosis. Am J Physiol Lung Cell Mol Physiol 313 (2017) L796-L806.

[9] R.L. Gieseck, 3rd, M.S. Wilson, and T.A. Wynn, Type 2 immunity in tissue repair and fibrosis. Nat Rev Immunol 18 (2018) 62-76. Medvedovic, S.R. Dillon, and S.K. Madala, IL-31-Driven Skin Remodeling Involves Epidermal Cell Proliferation and Thickening That Lead to Impaired Skin-Barrier Function. PLoS One 11 (2016) e0161877.

[11] S.R. Dillon, C. Sprecher, A. Hammond, J. Bilsborough, M. Rosenfeld-Franklin, S.R. 
Kuijper, T. Bukowski, P. Shea, D.L. Dong, M. Dasovich, F.J. Grant, L. Lockwood, produced by activated T cells, induces dermatitis in mice. Nature immunology 5 (2004) 752-60.

[12] E. Maier, D. Werner, A. Duschl, B. Bohle, and J. Horejs-Hoeck, Human Th2 but not Th9

[13] U. Raap, M. Gehring, S. Kleiner, U. Rudrich, B. Eiz-Vesper, H. Haas, A. Kapp, and B.F. Gibbs, Human basophils are a source of - and are differentially activated by - IL-31. Clin Exp Allergy 47 (2017) 499-508.

[14] N. Kunsleben, U. Rudrich, M. Gehring, N. Novak, A. Kapp, and U. Raap, IL-31 Induces Chemotaxis, Calcium Mobilization, Release of Reactive Oxygen Species, and CCL26 in Eosinophils, Which Are Capable to Release IL-31. J Invest Dermatol 135 (2015)

[15] Y. Yagi, A. Andoh, A. Nishida, M. Shioya, T. Nishimura, T. Hashimoto, T. Tsujikawa, Y. 1908-1911. Saito, and Y. Fujiyama, Interleukin-31 stimulates production of inflammatory mediators from human colonic subepithelial myofibroblasts. Int J Mol Med 19 (2007) induces cytokine and chemokine production from human bronchial epithelial cells through activation of mitogen-activated protein kinase signalling pathways: implications for the allergic response. Immunology 122 (2007) 532-41. 
[17] F. Cevikbas, X.D. Wang, T. Akiyama, C. Kempkes, T. Savinko, A. Antal, G. Kukova, T. Buhl, A. Ikoma, J. Buddenkotte, V. Soumelis, M. Feld, H. Alenius, S.R. Dillon, E. Carstens, B. Homey, A. Basbaum, and M. Steinhoff, A sensory neuron-expressed IL31 receptor mediates T helper cell-dependent itch: Involvement of TRPV1 and

[18] S. Oyama, H. Kitamura, T. Kuramochi, Y. Higuchi, H. Matsushita, T. Suzuki, M. Goto, H. Adachi, K. Kasutani, A. Sakamoto, Y. Iwayanagi, A. Kaneko, M. Nanami, E. Fujii, K. Esaki, Y. Takashima, S. Shimaoka, K. Hattori, and Y. Kawabe, Cynomolgus monkey model of interleukin-31-induced scratching depicts blockade of human

[19] K. Psachoulia, K.A. Chamberlain, D. Heo, S.E. Davis, J.D. Paskus, S.E. Nanescu, J.L. interleukin-31 receptor A by a humanized monoclonal antibody. Exp Dermatol 27 Dupree, T.A. Wynn, and J.K. Huang, IL4I1 augments CNS remyelination and axonal

513 [20] W. Lin, Q. Zhou, C. Liu, M. Ying, and S. Xu, Increased plasma IL-17, IL-31, and IL-33 (2018) 14-21. levels in chronic spontaneous urticaria. Sci Rep 7 (2017) 17797. protection by modulating T cell driven inflammation. Brain 139 (2016) 3121-3136.

[22] R. Edukulla, B. Singh, A.G. Jegga, V. Sontake, S.R. Dillon, and S.K. Madala, Th2 
[23] V. Sontake, R.K. Kasam, D. Sinner, T.R. Korfhagen, G.B. Reddy, E.S. White, A.G. Jegga, and S.K. Madala, Wilms' tumor 1 drives fibroproliferation and myofibroblast transformation in severe fibrotic lung disease. JCl Insight 3 (2018).

[24] S.K. Madala, R. Edukulla, K.R. Davis, S. Schmidt, C. Davidson, J.A. Kitzmiller, W.D. Hardie, and T.R. Korfhagen, Resistin-like molecule alpha1 (Fizz1) recruits lung dendritic cells without causing pulmonary fibrosis. Respiratory research 13 (2012) 51.

[25] S.K. Madala, S. Schmidt, C. Davidson, M. Ikegami, S. Wert, and W.D. Hardie, MEKERK pathway modulation ameliorates pulmonary fibrosis associated with epidermal growth factor receptor activation. Am J Respir Cell Mol Biol 46 (2012) 380-8.

[26] S.K. Madala, R. Edukulla, S. Schmidt, C. Davidson, M. Ikegami, and W.D. Hardie, Bone marrow-derived stromal cells are invasive and hyperproliferative and alter transforming growth factor-alpha-induced pulmonary fibrosis. Am J Respir Cell Mol

[27] R.K. Kasam, S. Ghandikota, D. Soundararajan, G.B. Reddy, S.K. Huang, A.G. Jegga, and S.K. Madala, Inhibition of Aurora Kinase B attenuates fibroblast activation and pulmonary fibrosis. EMBO Mol Med 12 (2020) e12131.

[28] S. Nobbe, P. Dziunycz, B. Muhleisen, J. Bilsborough, S.R. Dillon, L.E. French, and G.F. Hofbauer, IL-31 expression by inflammatory cells is preferentially elevated in atopic dermatitis. Acta Derm Venereol 92 (2012) 24-8.

539 [29] R.K. Kasam, P.R. Gajjala, A.G. Jegga, J.A. Courtney, S.H. Randell, E.L. Kramer, J.P. Clancy, and S.K. Madala, Fibrocyte accumulation in the lungs of cystic fibrosis patients. J Cyst Fibros 19 (2020) 815-822. 
542 [30] V. Sontake, S.K. Shanmukhappa, B.A. DiPasquale, G.B. Reddy, M. Medvedovic, W.D. Hardie, E.S. White, and S.K. Madala, Fibrocytes Regulate Wilms Tumor 1-Positive

[31] D.J.K. Yombo, M.M. Mentink-Kane, M.S. Wilson, T.A. Wynn, and S.K. Madala, Heat shock protein 70 is a positive regulator of airway inflammation and goblet cell hyperplasia in a mouse model of allergic airway inflammation. J Biol Chem 294 (2019) 15082-15094.

[32] S.K. Madala, R. Edukulla, M. Phatak, S. Schmidt, C. Davidson, T.H. Acciani, T.R. Korfhagen, M. Medvedovic, T.D. Lecras, K. Wagner, and W.D. Hardie, Dual targeting of MEK and PI3K pathways attenuates established and progressive pulmonary fibrosis. PloS one 9 (2014) e86536.

[33] J. Chen, E.E. Bardes, B.J. Aronow, and A.G. Jegga, ToppGene Suite for gene list enrichment analysis and candidate gene prioritization. Nucleic Acids Res 37 (2009) W305-11.

[34] V. Sontake, Y. Wang, R.K. Kasam, D. Sinner, G.B. Reddy, A.P. Naren, F.X. McCormack, E.S. White, A.G. Jegga, and S.K. Madala, Hsp90 regulation of fibroblast activation in pulmonary fibrosis. JCI Insight 2 (2017) e91454. Kauder, S. Biswas, S.K. Karnik, C. Ha, Z. Modrusan, M.A. Matthay, J. Kukreja, H.R. Collard, J.G. Egen, P.J. Wolters, and J.R. Arron, Heterogeneous gene expression signatures correspond to distinct lung pathologies and biomarkers of disease severity in idiopathic pulmonary fibrosis. Thorax 70 (2015) 48-56. 
[36] J.H. Ryu, T. Moua, C.E. Daniels, T.E. Hartman, E.S. Yi, J.P. Utz, and A.H. Limper, Idiopathic pulmonary fibrosis: evolving concepts. Mayo Clin Proc 89 (2014) 1130-42.

[37] T.A. Wynn, and T.R. Ramalingam, Mechanisms of fibrosis: therapeutic translation for fibrotic disease. Nat Med 18 (2012) 1028-40.

[38] H.J. Kim, M. Zeidi, D. Bonciani, S.M. Pena, J. Tiao, S. Sahu, and V.P. Werth, Itch in dermatomyositis: the role of increased skin interleukin-31. Br J Dermatol 179 (2018) 669-678.

[39] D. Ming, X. Yu, R. Guo, Y. Deng, J. Li, C. Lin, M. Su, Z. Lin, and Z. Su, Elevated TGFbeta1/IL-31 Pathway Is Associated with the Disease Severity of Hepatitis B VirusRelated Liver Cirrhosis. Viral Immunol 28 (2015) 209-16. Chen, C. Pothoulakis, H.W. Koon, and S.R. Targan, Inhibition of a novel fibrogenic factor Tl1a reverses established colonic fibrosis. Mucosal Immunol 7 (2014) 1492-

[41] B. Yaseen, H. Lopez, Z. Taki, S. Zafar, H. Rosario, B.A. Abdi, S. Vigneswaran, F. Xing, N. Arumalla, S. Black, S. Ahmad, K. Kumar, R. Gul, L. Scolamiero, S. Morris, A. 503.

583 [42] E.A. Ayaub, A. Dubey, J. Imani, F. Botelho, M.R.J. Kolb, C.D. Richards, and K. Ask, Overexpression of OSM and IL-6 impacts the polarization of pro-fibrotic macrophages and the development of bleomycin-induced lung fibrosis. Sci Rep 7 (2017) 13281. 
[43] G.S. Wong, J.L. Redes, N. Balenga, M. McCullough, N. Fuentes, A. Gokhale, C. Koziol-White, J.A. Jude, L.A. Madigan, E.C. Chan, W.H. Jester, S. Biardel, N. Flamand, R.A. Panettieri, Jr., and K.M. Druey, RGS4 promotes allergen- and aspirinassociated airway hyperresponsiveness by inhibiting PGE2 biosynthesis. J Allergy Clin Immunol (2020).

[44] J. Huang, H. Yue, T. Jiang, J. Gao, Y. Shi, B. Shi, X. Wu, and X. Gou, IL-31 plays dual roles in lung inflammation in an OVA-induced murine asthma model. Biol Open 8 (2019).

[45] M.A. Thomas, B.J. Buelow, A.M. Nevins, S.E. Jones, F.C. Peterson, R.L. Gundry, M.H. Grayson, and B.F. Volkman, Structure-function analysis of CCL28 in the development of post-viral asthma. J Biol Chem 290 (2015) 4528-36.

[46] N. Weathington, M.E. O'Brien, J. Radder, T.C. Whisenant, E.R. Bleecker, W.W. Busse, S.C. Erzurum, B. Gaston, A.T. Hastie, N.N. Jarjour, D.A. Meyers, J. Milosevic, W.C. Moore, J.R. Tedrow, J.B. Trudeau, H.P. Wong, W. Wu, N. Kaminski, S.E. Wenzel, and B.D. Modena, BAL Cell Gene Expression in Severe Asthma Reveals 


\section{Figure $1 \quad$ IL-31RA}

A

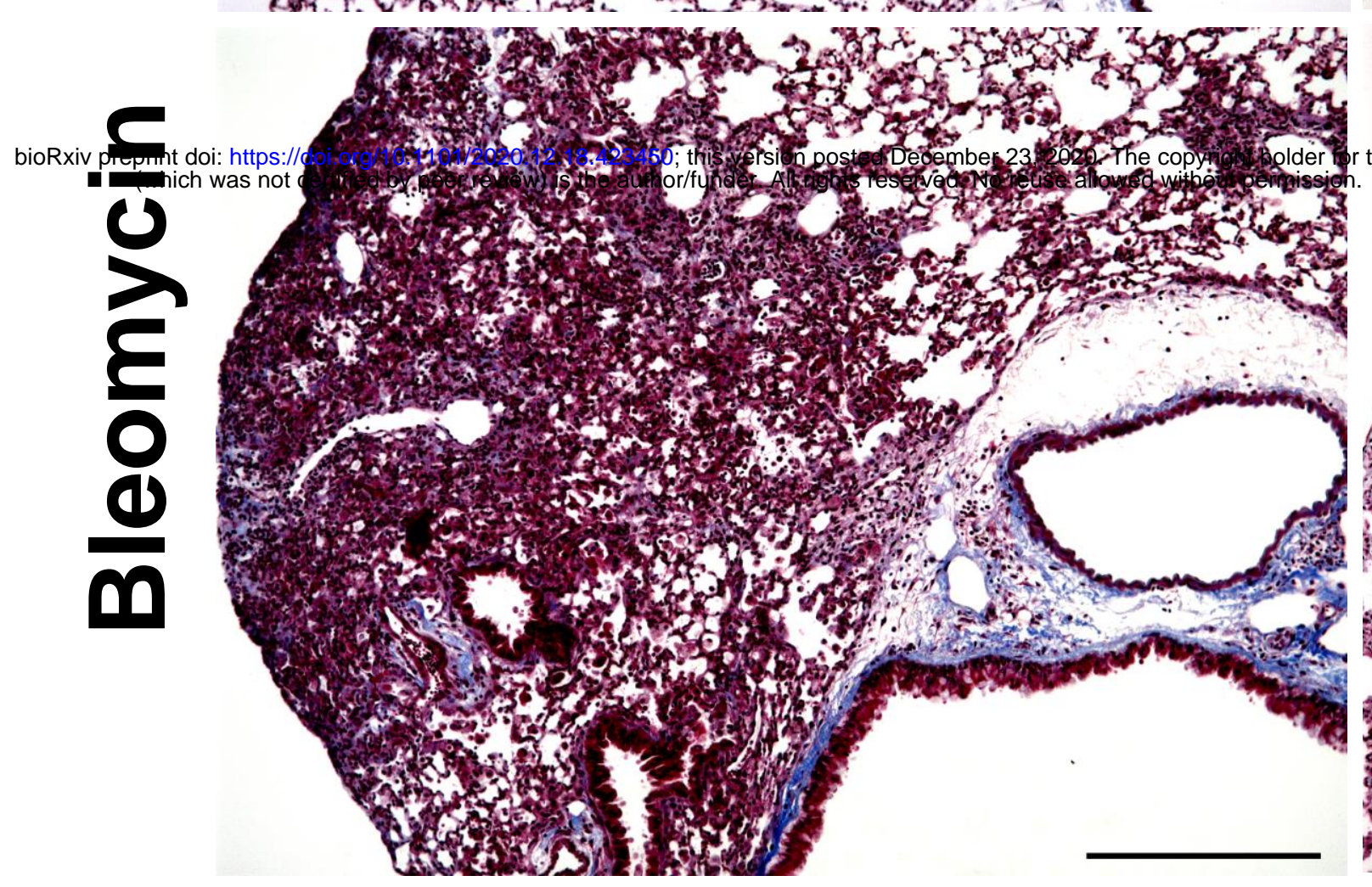

C

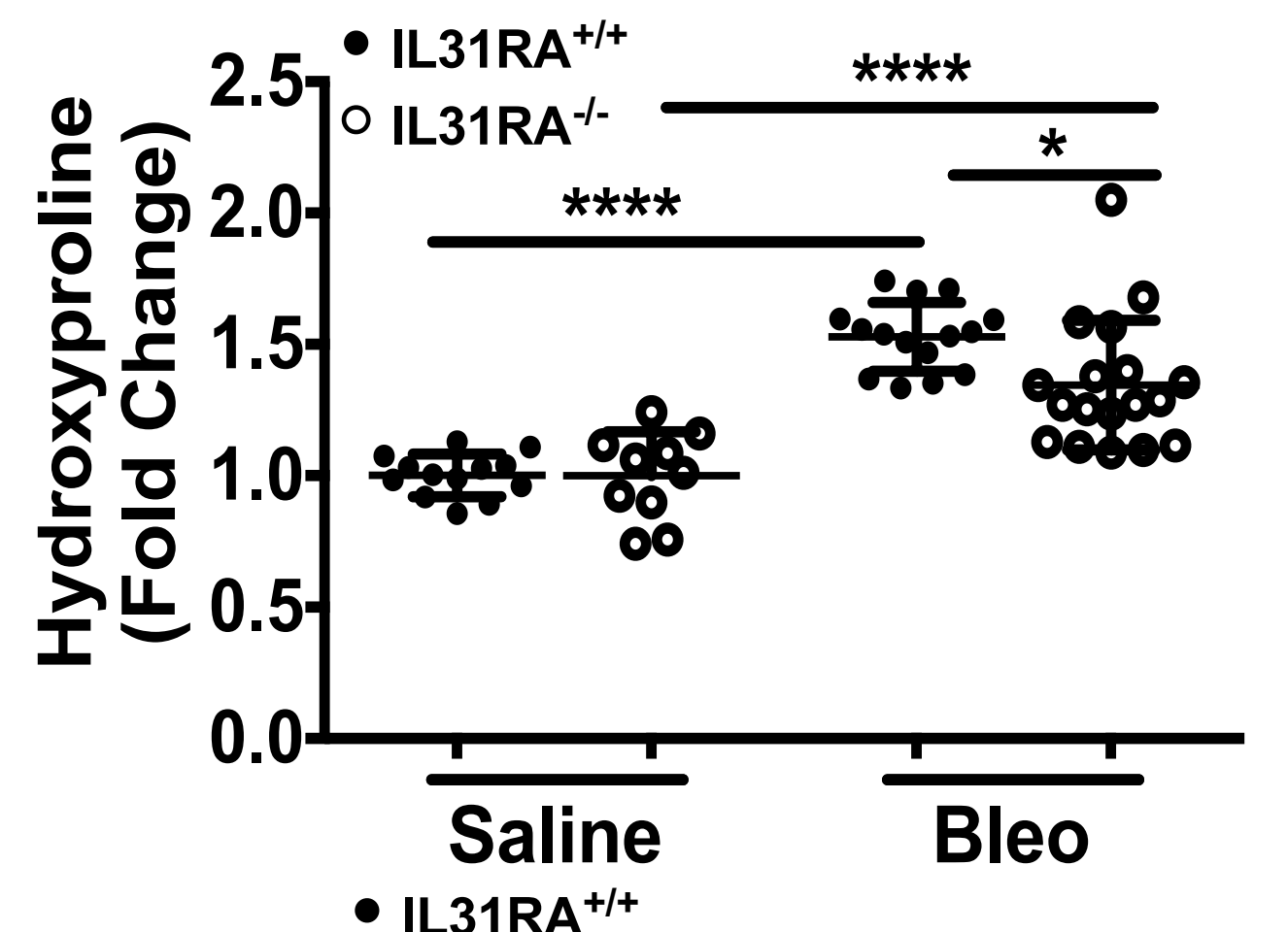

E

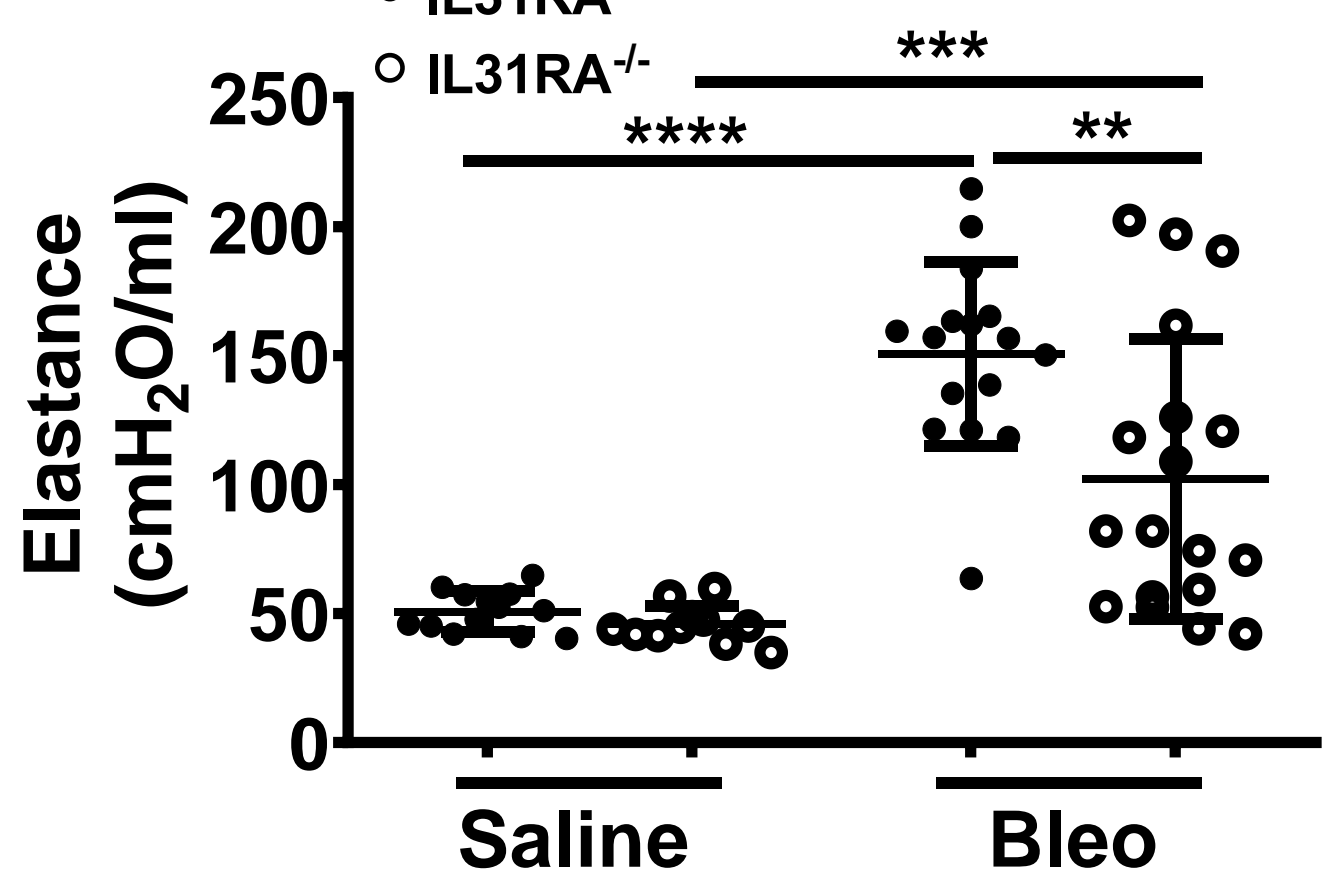

(L-31RA

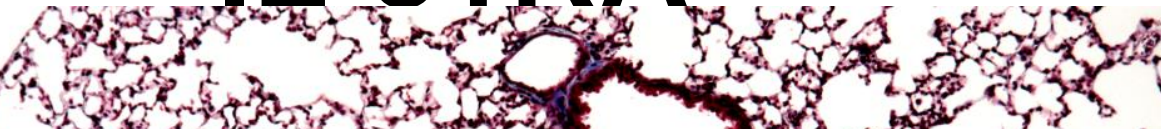

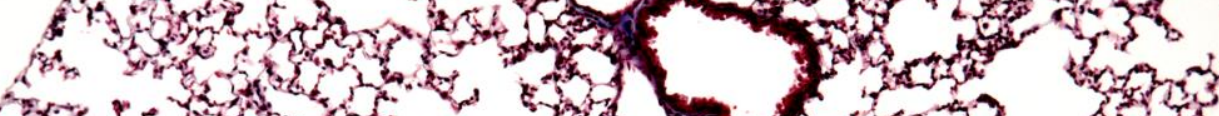
6 theng

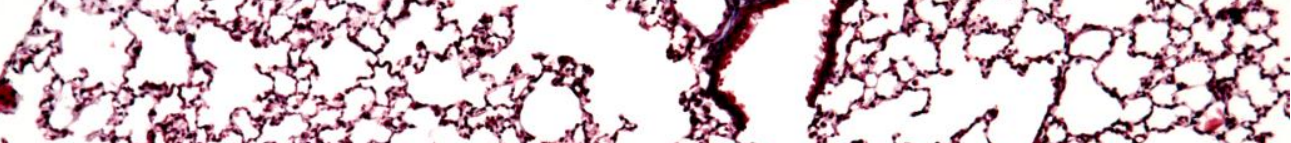

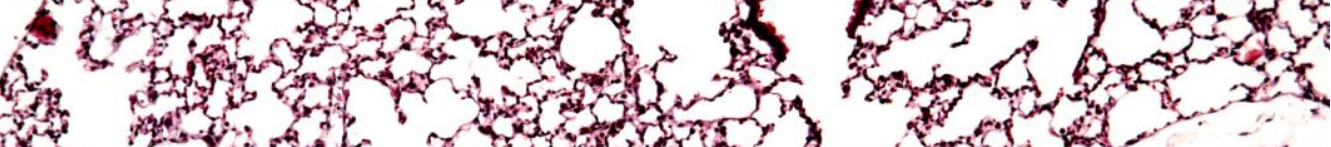
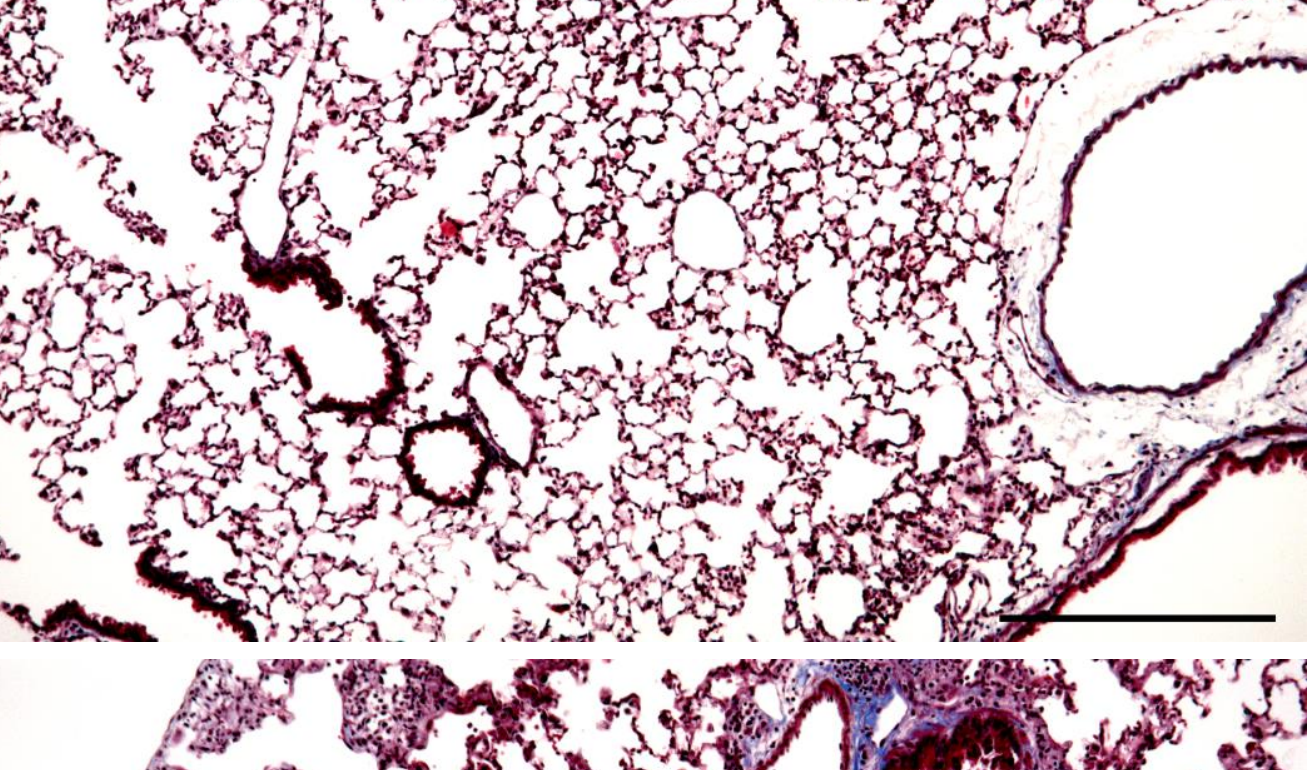

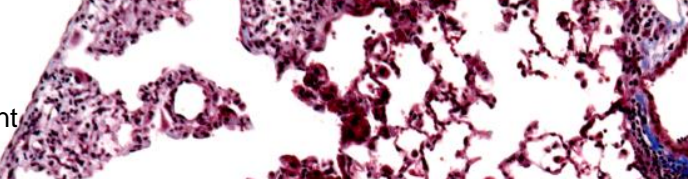
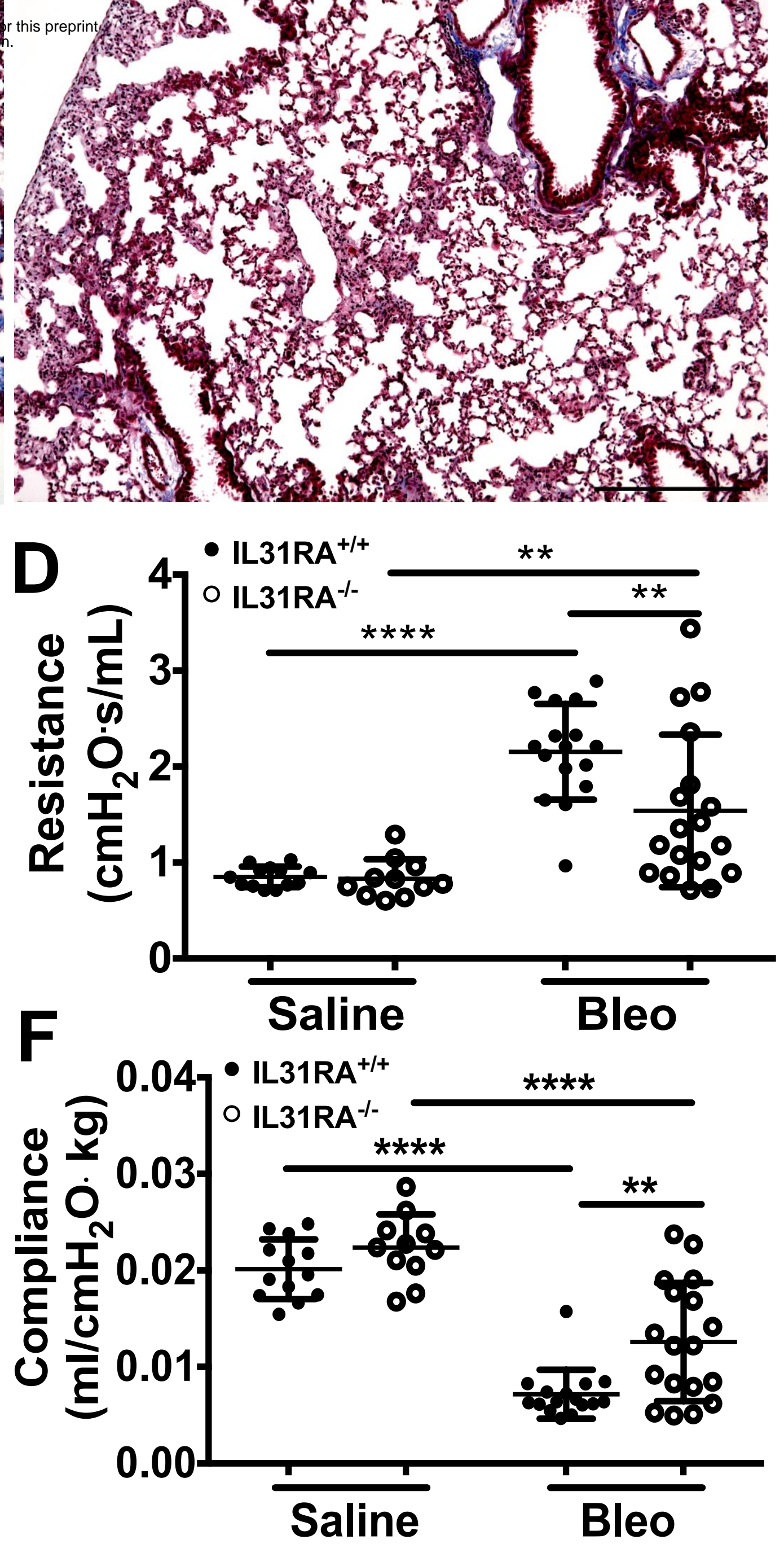
A
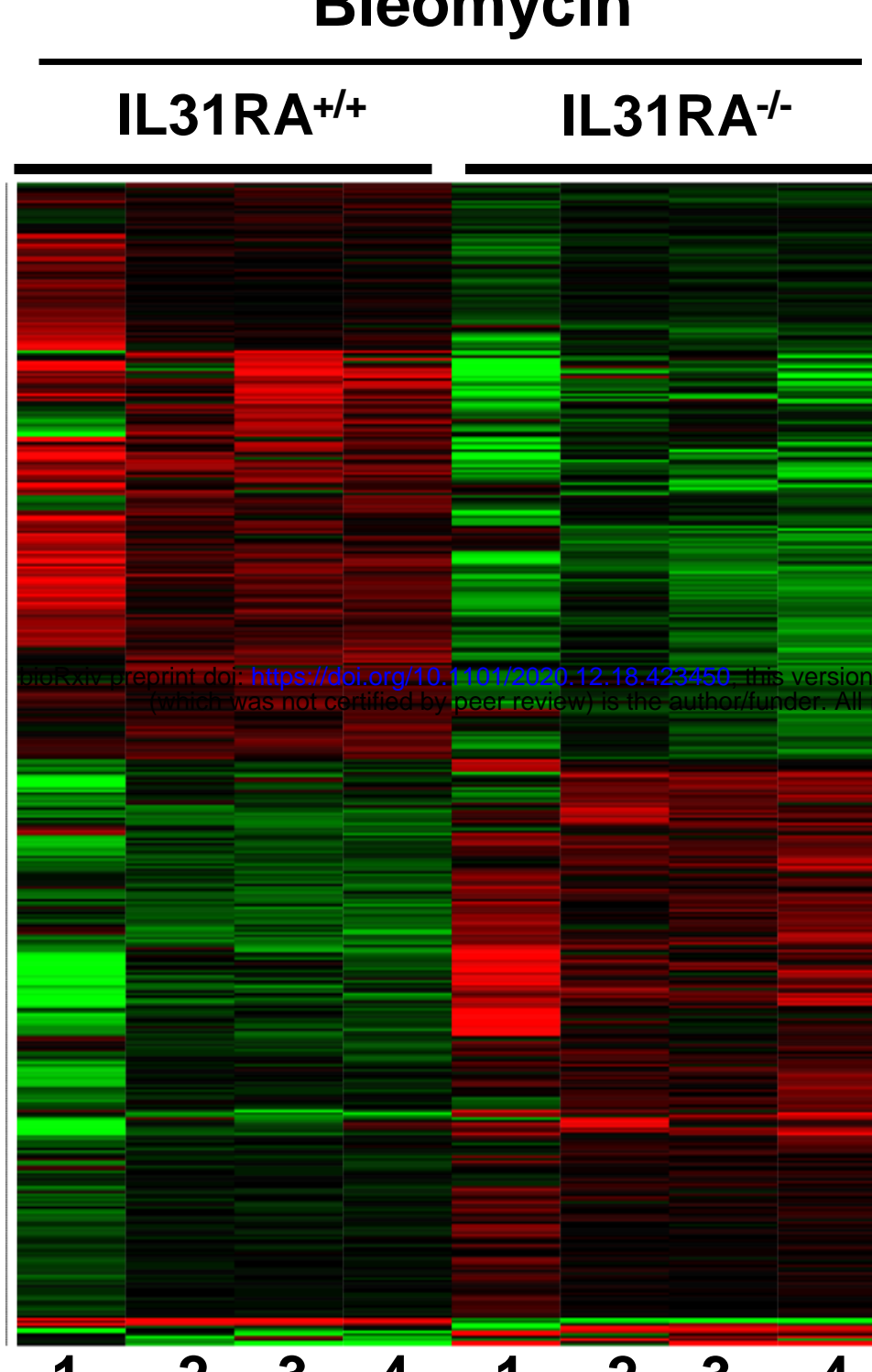

$\begin{array}{llllllll}1 & 2 & 3 & 4 & 1 & 2 & 3 & 4\end{array}$ Color Key \begin{tabular}{llll}
\hline-1 & 0 & 1
\end{tabular}

B

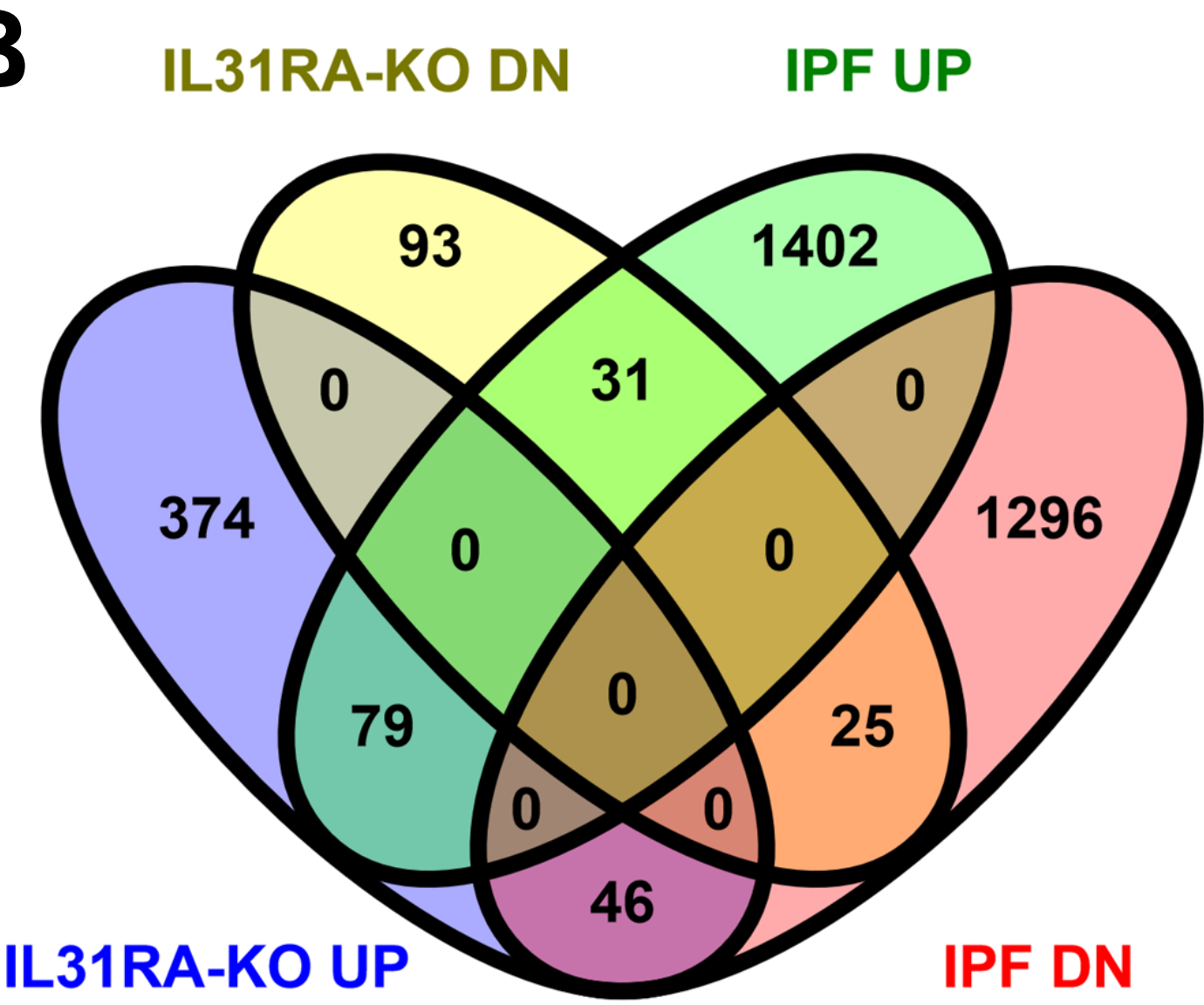

C
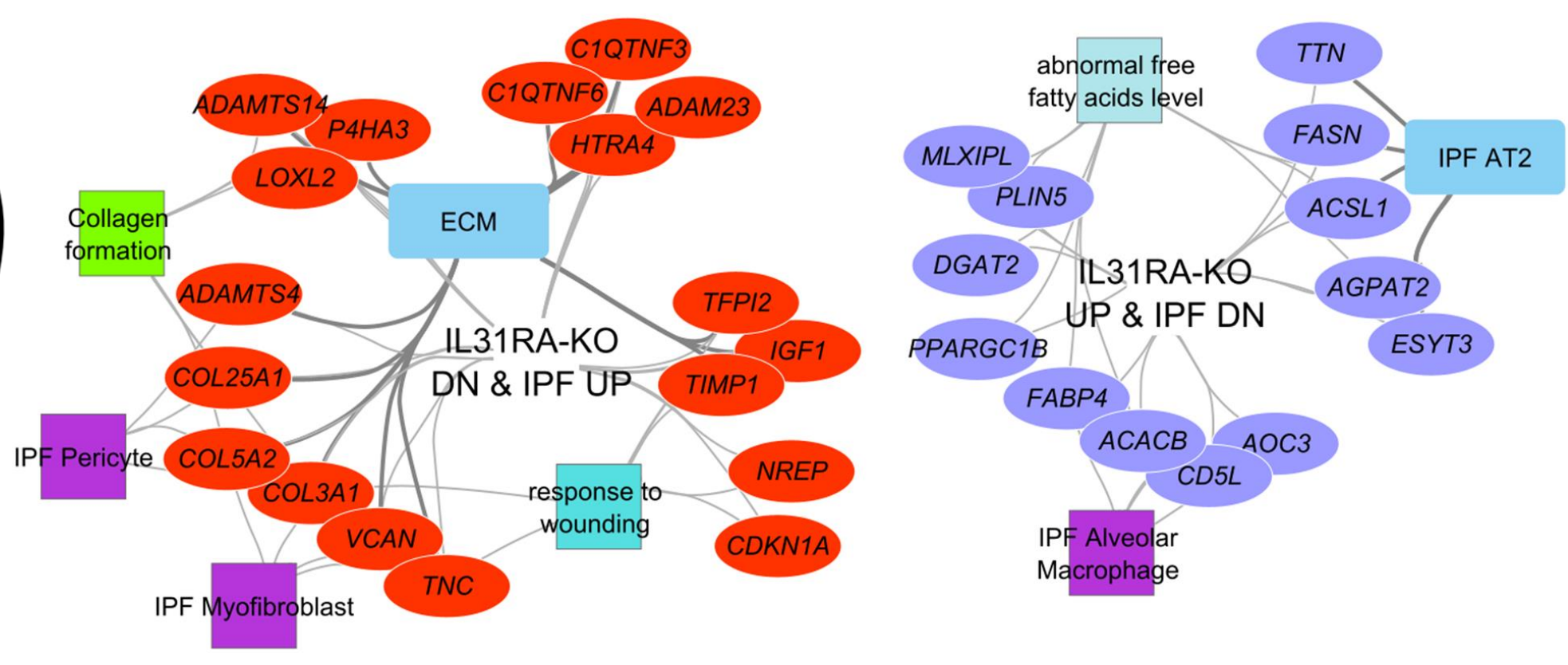

\section{ECM - related genes}

A2m, Arg1, Mmp13, Col1a1, Col3a1, Col5a2,Timp1, Eln, Nrg1, Ereg ,Fn1, IL6 G a(i) signaling

Ccl28, Rgs5,Rgs4,Rgs16, Ptgdr2

Epithelium-associated genes

Nrg1, Krt20, Krt4, Krt5, Krt14, Sfrp1, Krt17,

Chemotaxis

Ccl28, Mcp1, II6, Cxc/13

Adenylate cyclase activity

Calcb, Calcr, gper1, mtnr1a, Ptgdr2

Up in IL31RA ${ }^{-/-}$mice GO enrichment (example genes)

\section{requlation of membrane potential}

Avpr1a, Adra1a ,Kcna2, Kcnu1, Alox12, dpp6, gria1,Grik4, Rgs7, Kcnh4, Grip2 $\mathrm{G}$ protein-coupled receptor binding

Avpr1a, Adra2a, Agtr1, Gria1, Edn3, Tff2, Vipr2

Synaptic signaling

Avpr1a, Nrn1, Colq, Adra1a, Adra2a, Kcna2, Nr3c2, gria1, grik4 ,slc1a2, edn3, positive requlation of cytosolic calcium ion concentration

Avpr1a, Adra1a, Agtr1, Ccr3, Ric3, Gpr18, Gpr33, Snca,Cxcr1

epithelial cell-associated genes

Chi3/1, clca1, Krt23, lamp3, Krt79 


\section{Figure 3}

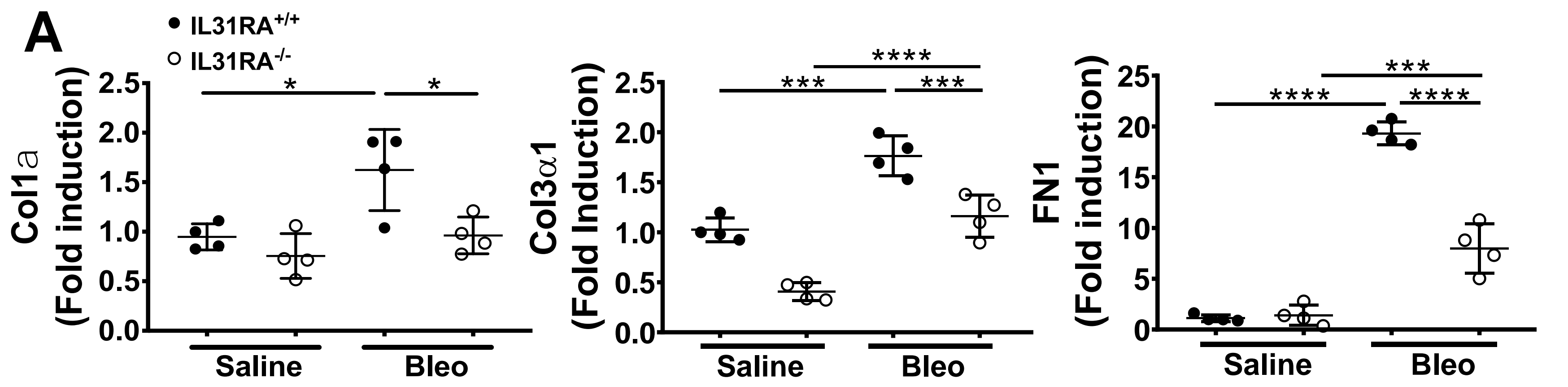

bioRxiv preprint doi: https:///doi.org/10.1101/2020.12.18.423450; this version posted December 23, 2020. The copyright holder for this preprint

B
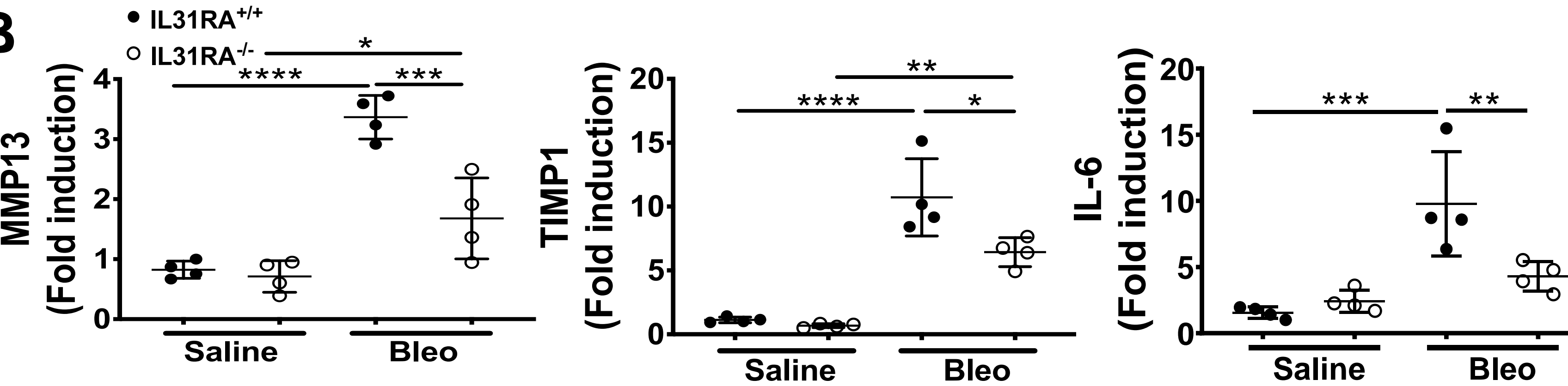
Figure 4

A

- IL31RA ${ }^{+/+}$

o IL31RA ${ }^{-/-}$

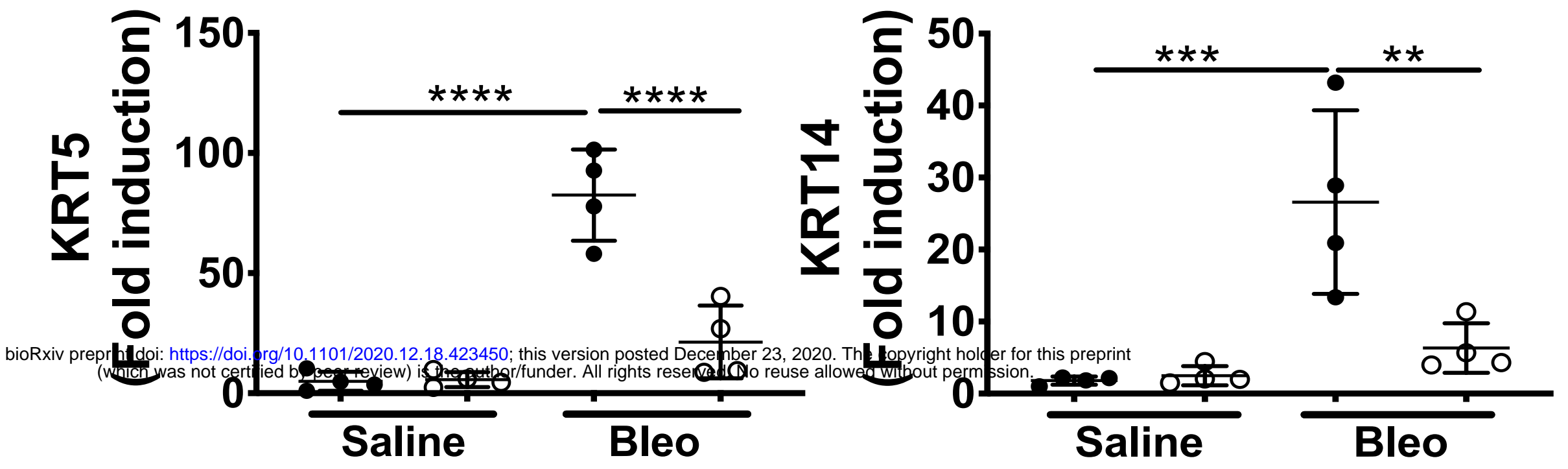

B
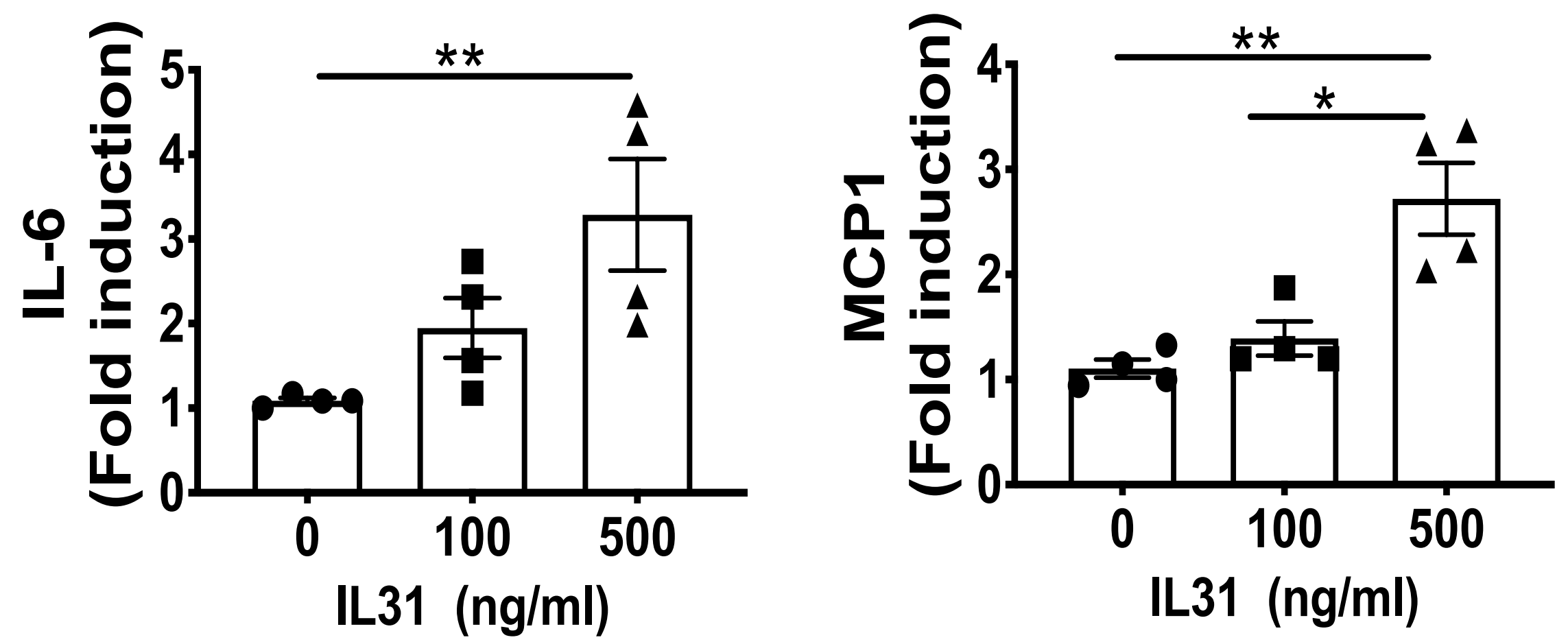

C
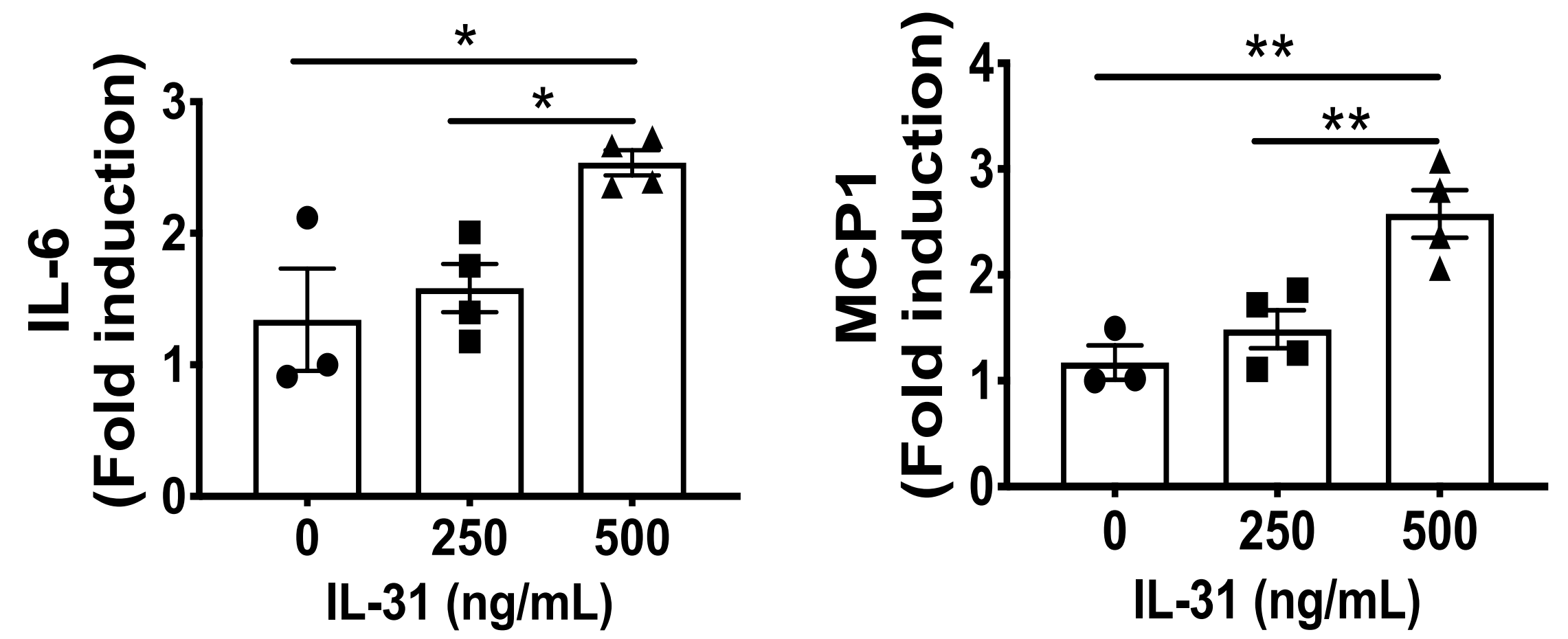
Figure 5

A

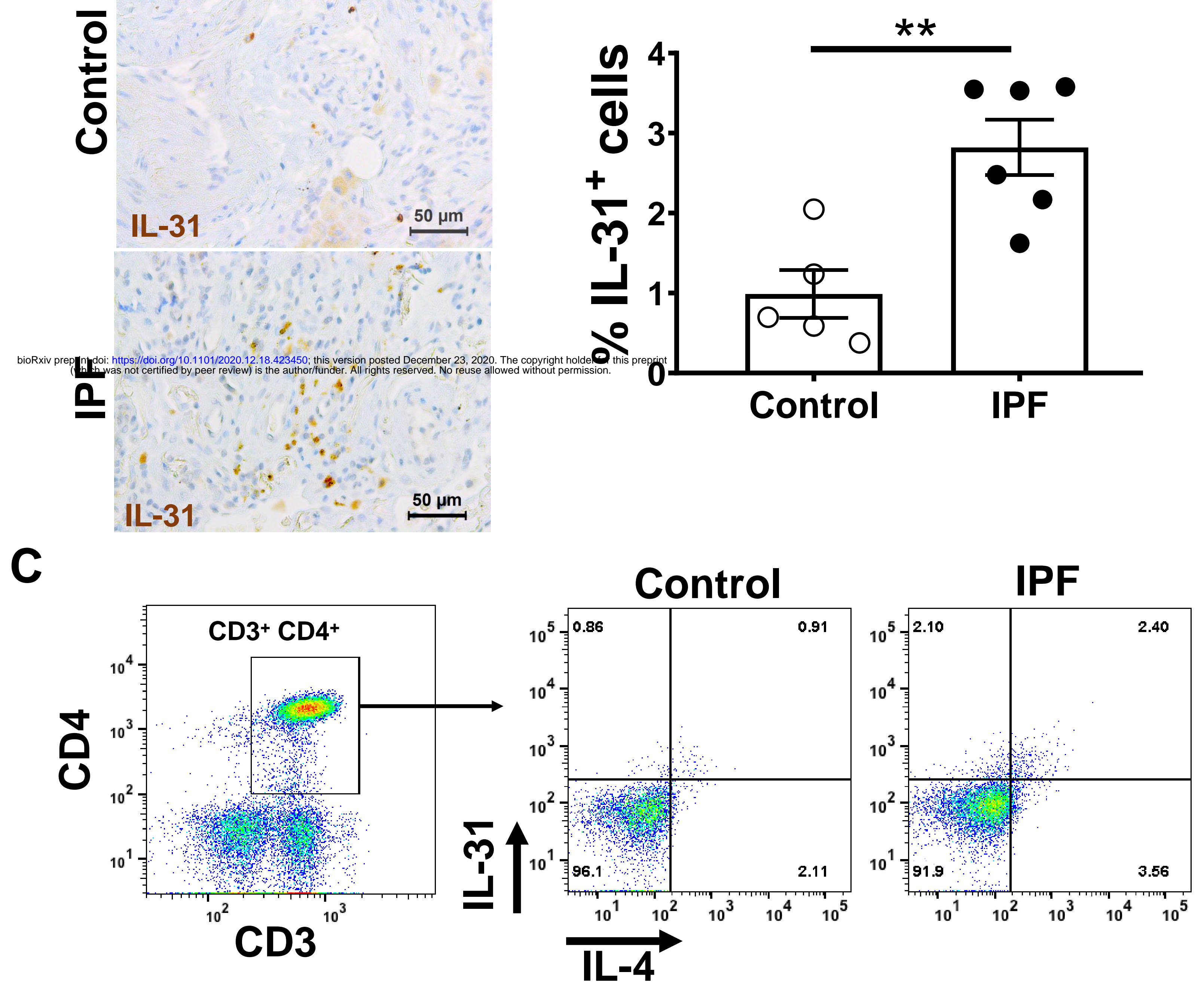

D

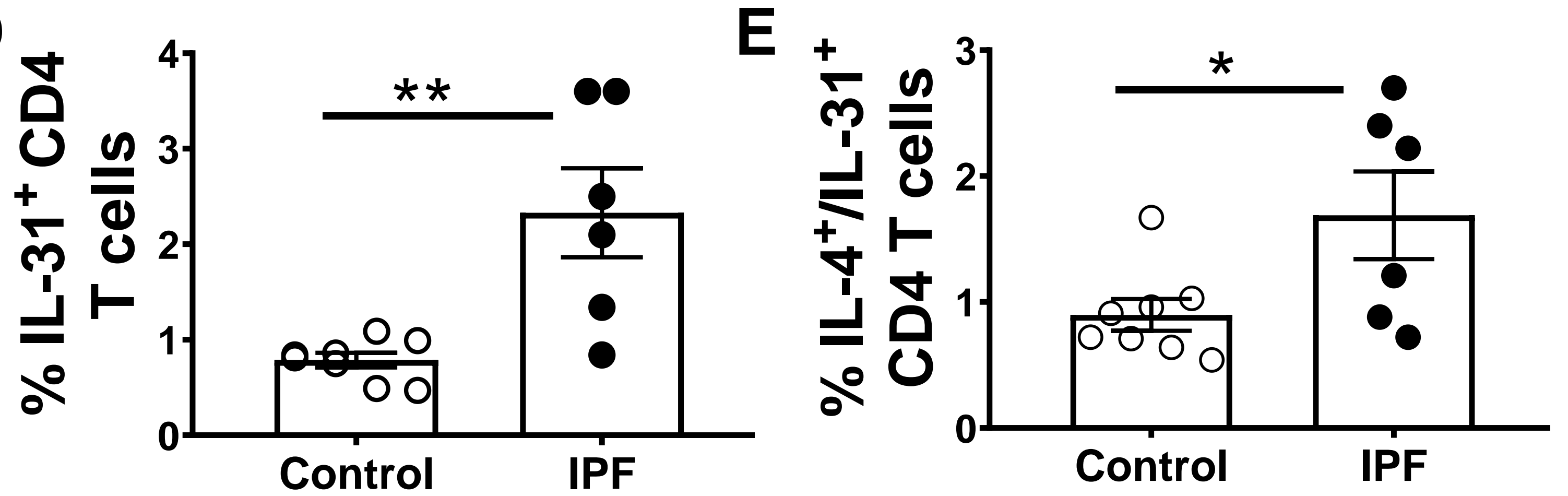

